\title{
Correction to: Age-specific reference values and cut-off points for anti-müllerian hormone in infertile women following a long agonist treatment protocol for IVF
}

\author{
Z. Heidar ${ }^{1} \cdot$ M. Bakhtiyari $^{2,3} \cdot$ F. Foroozanfard ${ }^{4} \cdot$ M. $^{\text {Mirzamoradi }}{ }^{5}$
}

Published online: 20 July 2018

(C) Italian Society of Endocrinology (SIE) 2018

\section{Correction to: Journal of Endocrinological Investigation (2018) 41:773-780 \\ https://doi.org/10.1007/s40618-017-0802-z}

Unfortunately, the first author name was incorrectly published in the original publication. The complete correct name should read as follows.

Zahra Heidar

The original article can be found online at https://doi.org/10.1007/ s40618-017-0802-z.

M. Mirzamoradi

dr.moradi000@yahoo.com

1 Infertility and Reproductive Health Research Center (IRHRC), Shahid Beheshti University of Medical Sciences, Tehran, Iran

2 Non-communicable Disease Research Center, Alborz University of Medical Sciences, Karaj, Iran

3 Department of Epidemiology and Biostatistics, School of Public Health, Tehran University of Medical Sciences, Tehran, Iran

4 Kashan University of Medical Sciences, Kashan, Iran

5 Department of Obstetrics and Gynecology, Mahdiyeh Hospital, Shahid Beheshti University of Medical Sciences, Po.Box: 1185817311, Tehran, Iran 